•技术与方法・

\title{
被动声学监测技术在西黑冠长臂猿监测中的应用
}

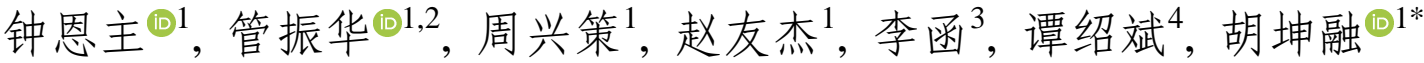

1. 西南林业大学大数据与智能工程学院, 昆明 650224; 2. 西南林业大学云南生物多样性研究院, 昆明 $650224 ; 3$. 哀牢山国家级自然保护

区，云南楚雄 675000; 4. 楚雄彝族自治州森林和草原资源监测站, 云南楚雄 675000

摘要: 近年来, 被动声学监测技术被广泛应用于陆生哺乳动物的监测, 它能以较低的价格和非侵入的方式在特定区域进行野 生动物无人值守监测, 面临的主要问题是需要人工收回数据和后期数据分析整理较为困难。本研究设计了一套被动声学监测 系统用于西黑冠长臂猿(Nomascus concolor)监测, 监测系统在野外由太阳能供电, 使用自研的指向性拾音器阵列采集鸣声数 据, 并通过无线网桥实时传输数据至管护局办公楼的服务器进行存储, 通过后台的鸣声数据管理系统辅助研究人员识别鸣声 和辨认鸣声来源方向, 简化数据采集和处理流程。该系统在哀牢山国家级自然保护区枇杷等科研监听点对两个西黑冠长臂猿 群体进行了 351 天的连续监测, 特点为: 系统运行长期稳定, 数据通过无线方式传输便捷高效且不受季节天气影响。指向性拾 音器阵列能有效分辨长臂猿鸣声来源方向, 弥补了传统监测设备难以分辨鸣声方向的缺陷。该系统与现有人工监测方法相比 在数据采集的持续性、连续性、完整度以及鸣声数据处理智能化和监测成本方面均具有一定优势, 符合西黑冠长臂猿持续长 期监测需求, 未来可作为西黑冠长臂猿自动化监测的解决方案进行推广应用。

关键词: 被动声学监测; 指向性拾音器阵列; 西黑冠长臂猿; 声源定位

钟恩主, 管振华, 周兴策, 赵友杰, 李函, 谭绍斌, 胡坤融 (2021) 被动声学监测技术在西黑冠长臂猿监测中的应用. 生物多样性, 29, 109-117. doi: 10.17520/biods.2020215.

Zhong EZ, Guan ZH, Zhou XC, Zhao YJ, Li H, Tan SB, Hu KR (2021) Application of passive acoustic monitoring technology in the monitoring of western black crested gibbons. Biodiversity Science, 29, 109-117. doi: 10.17520/biods.2020215.

\section{Application of passive acoustic monitoring technology in the monitoring of western black crested gibbons}

\author{
Enzhu Zhong ${ }^{\circledR 1}$, Zhenhua Guan ${ }^{\circledR 1,2}$, Xingce Zhou ${ }^{1}$, Youjie Zhao ${ }^{1}$, Han $\mathrm{Li}^{3}$, Shaobin $\mathrm{Tan}^{4}$, Kunrong $\mathrm{Hu}^{\circledR 1 *}$ \\ 1 College of Big Data and Intelligent Engineering, Southwest Forestry University, Kunming 650224 \\ 2 Yunnan Academy of Biodiversity, Southwest Forestry University, Kunming 650224 \\ 3 Ailao Mountain National Nature Reserve, Chuxiong, Yunnan 675000 \\ 4 Monitoring Station of Forest and Grassland Resources in Chuxiong Yi Autonomous Prefecture, Chuxiong, Yunnan 675000
}

\begin{abstract}
Aims: Passive acoustic monitoring technology has begun to be widely used for monitoring terrestrial mammals. Such technology enables the unattended monitoring of wildlife in specific areas at a low-cost and in a non-invasive manner. The main problem, however, is the need to manually retrieve the data. Moreover, the subsequent data analysis and sorting can be complicated.

Innovation: In this study, a passive acoustic monitoring system was designed for monitoring the western black crested gibbon (Nomascus concolor). The monitoring system is powered by solar energy in the field. Sound data are collected by a directional pick-up array. The data are transmitted through a wireless network to a server in the management office building. Researchers are assisted in identifying the sound and the direction of the sound by using the sound-data management system, which simplifies data collection and processing.
\end{abstract}

收稿日期: 2020-05-26; 接受日期: 2020-07-31

基金项目: 国家自然科学基金(31960142)、云南省教育厅科学研究基金(2017ZZX212)、云南省专业学位研究生教学案例库项目和云南省高校优势特色 重点学科(生物学)建设项目

* 通讯作者 Author for correspondence. E-mail: hukunrong@swfu.edu.cn 
Significance: This system has been continuously monitoring two groups of western black crested gibbons for at least 351 days at the monitoring site (of Pipaqing in the Ailao Mountain Nature Reserve). Monitoring results up until now demonstrate that the system has had a long continuous run-time and is not affected by the weather conditions; data transmission is convenient and efficient; the directions of sound source can be determined by the directional pick-up array well, overcoming the shortcomings of traditional monitoring equipment. This system has advantages over manual monitoring methods in terms of data continuity and integrity, intelligence in data processing, and monitoring costs. This system meets the continuous long-term monitoring needs of western black crested gibbons, and can be improved and applied in the future as an automated solution for the monitoring of this primate species.

Key words: passive acoustic monitoring; directional pick-up array; western black crested gibbon; sound source localization

对野生动物进行长期监测是野生动物研究、保 护、管理和资源可持续利用的核心环节。被动声学 监测(passive acoustic monitoring, PAM)技术最早被 应用于水生动物的监测(Watkins \& Schevill, 1972)。 近年来, 该项技术也被应用到陆栖动物的监测中 (Blumstein et al, 2011), 包括昆虫(Chesmore \& Ohya, 2004)、鸟类 (Swiston \& Mennill, 2009) 以及哺乳动物 中的非洲象(Loxodonta africana, Thompson et al, 2010)、梅花鹿(Cervus nippon, Enari et al, 2017)、蝙 蝠(MacSwiney et al, 2008)、灵长类(Kalan et al, 2015, 2016)等。被动声学监测技术通过在野生动物活动区 域部署声学传感器记录动物鸣声, 以非侵入的方式 获取长期监测数据, 能有效弥补人工监测在数据获 取持续性和完整度方面的局限性, 同时降低时间成 本和人工开支。生态信息学和大数据技术的发展为 监测数据处理提供了更高效的手段, 但自动化的被 动声学监测技术目前仍处于探索阶段。

在众多具有鸣叫习性的哺乳动物中, 长臂猿科 动物鸣声独特且传播距离较远(范朋飞等, 2010), 鸣 叫通常被用于野生长臂猿种群的分布和数量调查, 以帮助制定合理的保护措施 (Brockelman \& Srikosamatara, 1993), 因此长臂猿十分适合作为被 动声学监测系统的测试对象。长臂猿科物种是小型 类人猿, 目前全球已被命名的 20 种长臂猿中, 有 6 种分布在中国海南、云南、广西的热带或亚热带森 林中, 4种被列为极危(CR, IUCN, 2020)。其中西黑 冠长臂猿(Nomascus concolor)在中国境内分布有最 大种群数量, 约270群(管振华等, 2017)。鸣叫是西黑 冠长臂猿的典型行为特征, 所有成年长臂猿个体都 能发出嘹亮的鸣声, 这些鸣声结构复杂, 具有个 体、性别、甚至群体差异(范朋飞等, 2010)。

迄今为止, 多项关于西黑冠长臂猿鸣声的研究
成果均是基于人工监测(Fan et al, 2009; Sun et al, 2011)。人工监测多以项目形式展开, 项目一旦结束 监测很难继续进行, 因此数据获取的持续性、完整 性能力不足(管振华等, 2017)。而现 有生物声学记录 设备的工作方式与红外相机类似, 需定期更换电池 及存储介质, 后期维护成本高, 且存在数据丢失及 损坏风险, 难以适应长期监测需求。同时, 当前监 测方法大多使用全向拾音器(Spillmann et al, 2015) 对长臂猿鸣叫方位进行判定, 监测时需要至少 3 台 能进行时间同步的设备, 按照三角形的方式部署在 长臂猿群体家域范围内进行基本的鸣声数据采集。 而后通过人工定期回收数据, 并使用专业声学软件 进行汇总计算才能判定鸣声来源方向, 一旦有一台 设备出现故障或是监测设备部署不合理便无法实 现鸣叫方位判定。整体操作复杂且后期管理成本较 高, 更多的是在短期监测项目中使用。

为满足西黑冠长臂猿长期监测需求, 本研究设 计了一套自动化被动声学监测系统。监测系统由太 阳能供电, 使用自研的指向性拾音器阵列对西黑冠 长臂猿进行监测, 并利用无线传输系统将监测到的 鸣声数据实时传输至管护局中心机房服务器存储, 配合数据分析管理系统, 方便研究人员随时管理和 分析监测数据。

\section{材料与方法}

\section{1 实验地点}

哀牢山国家级自然保护区楚雄片区位于哀牢 山中北段山体的东面, 地理位置为 $100^{\circ} 54^{\prime}-$ $101^{\circ} 30^{\prime} \mathrm{E}, 23^{\circ} 36^{\prime}-24^{\circ} 44^{\prime} \mathrm{N}$, 总面积为 $46.56 \mathrm{~km}^{2}$, 占 整个云南哀牢山国家级自然保护区总面积 $\left(677 \mathrm{~km}^{2}\right)$ 的6.8\%。其中, 核心区面积为 $12.31 \mathrm{~km}^{2}$, 实验区为 $8.76 \mathrm{~km}^{2}$, 缓冲区为 $25.49 \mathrm{~km}^{2}$ 。主要保护对象是亚 
热带中山湿性常绿阔叶林及其生态系统, 以及西黑 冠长臂猿、红豆杉(Taxus chinensis)等珍稀濒危动植 物资源及其栖息地。

监测系统搭建于云南哀牢山国家级自然保护 区楚雄片区枇杷等科研监听点 $\left(101^{\circ} 02^{\prime} \mathrm{E}, 24^{\circ} 34^{\prime} \mathrm{N}\right.$, 孙国政等, 2012), 监听点海拔2,397 m, 地处保护区 核心区，四周空旷，利于长臂猿鸣声监听。

\section{2 系统设计}

\subsection{1 整体设计方案}

本研究设计的被动声学监测系统由 1 个监测点 和 2 个信号传输中继点组成, 每个点位均配备了相 同规格的太阳能供电系统和无线传输系统, 而指向 性拾音器阵列则仅在监测点配备, 数据管理系统部 署在管护局服务器(图1)。太阳能电池板单独安装在 离地 $2 \mathrm{~m}$ 的支架上, 其余设备均安装在小型三角拉 线监测塔上, 监测塔全高为 $10 \mathrm{~m}$ (塔身高 $8 \mathrm{~m}$, 避雷 针高 $2 \mathrm{~m}$ )。

\subsection{2 各部分设计}

(1)指向性拾音器阵列

本研究使用自研的指向性拾音器阵列(专利号: ZL 20182 2264510.6)进行鸣声数据采集。指向性拾 音器阵列由多个指向性拾音器按一定规则在同一 平面排列组成, 设计时为排除拾音器间距较小造成 的电磁干扰和空间混叠效应, 根据被监测声源最高 频率按公式1 (McCowan, 2001)对拾音器阵列中各拾 音器间隔进行设计。

$$
d \geq \frac{\lambda \min }{2}=\frac{c}{2 f}
$$

式中, $d$ 为拾音器间隔, 单位 $m ; \lambda$ 为波长, 单位 $m ; c$
为声速，空气中约为 $340 \mathrm{~m} / \mathrm{s} ; f$ 为频率，单位 $\mathrm{Hz}$ 。西 黑冠长臂猿鸣声频率主要集中于 $0.5-2 \mathrm{kHz}$ 左右，一 些强调音节谐波频率甚至可达到 $5.828 \mathrm{kHz}$ (范朋飞 等, 2010)。为便于计算, 本文将西黑冠长臂猿最高 鸣声频率记为 $6 \mathrm{kHz}$ ，带入公式(1), 计算得到用于 监测西黑冠长臂猿的指向性拾音器阵列内各拾音 器之间的空间距离不小于 $2.8 \mathrm{~cm}$ 即可。而本研究实 际制作时使用 6 只单独的指向性拾音器 (快鱼 TRADIO R系列户外拾音器, 监听范围 $45^{\circ}$ 夹角, 灵 敏度-38 dB, 信噪比85 dB)组成拾音器阵列, 考虑 到设备自身尺寸及安装维护等因素, 将拾音器间距 设计为 $30 \mathrm{~cm}$, 则真实的拾音器阵列排列近似一个 边长 $30 \mathrm{~cm}$ 的正六边形，六边形的 6 个顶点分别对应 6 只指向性拾音器(图2), 拾音器阵列监测频率范围 为 $20 \mathrm{~Hz}-22 \mathrm{kHz}$ ，除监测长臂猿外还适用于其他动 物的鸣声监测。

拾音器阵列安装在监测塔顶部(高度: $8 \mathrm{~m}$ ), 同 时各拾音器均安装了防风罩以减小风噪影响。阵列 内各拾音器按顺时针方向依次编号，其中3号拾音 器所指中心方向为西偏北 $20^{\circ}, 6$ 号拾音器所指中心 方向为东偏南 $20^{\circ}$ (图2a)。拾音器采集到的音频数据 存储格式为aac (采样率32 kHz, 位深: 32 bit, 单声 道), 每个文件存储 $30 \mathrm{~min}$ 音频数据, 大小为 10.3 $M B$, 音频文件按拾音器编号分别存储, 且每份音 频文件尾部均有拾音器编号标识，具体的音频文件 命名格式为“年_月_日_时_分_秒_拾音器编 号.aac”。

\section{(2)太阳能供电系统}

监测系统中除管护局外其余传输节点均由太

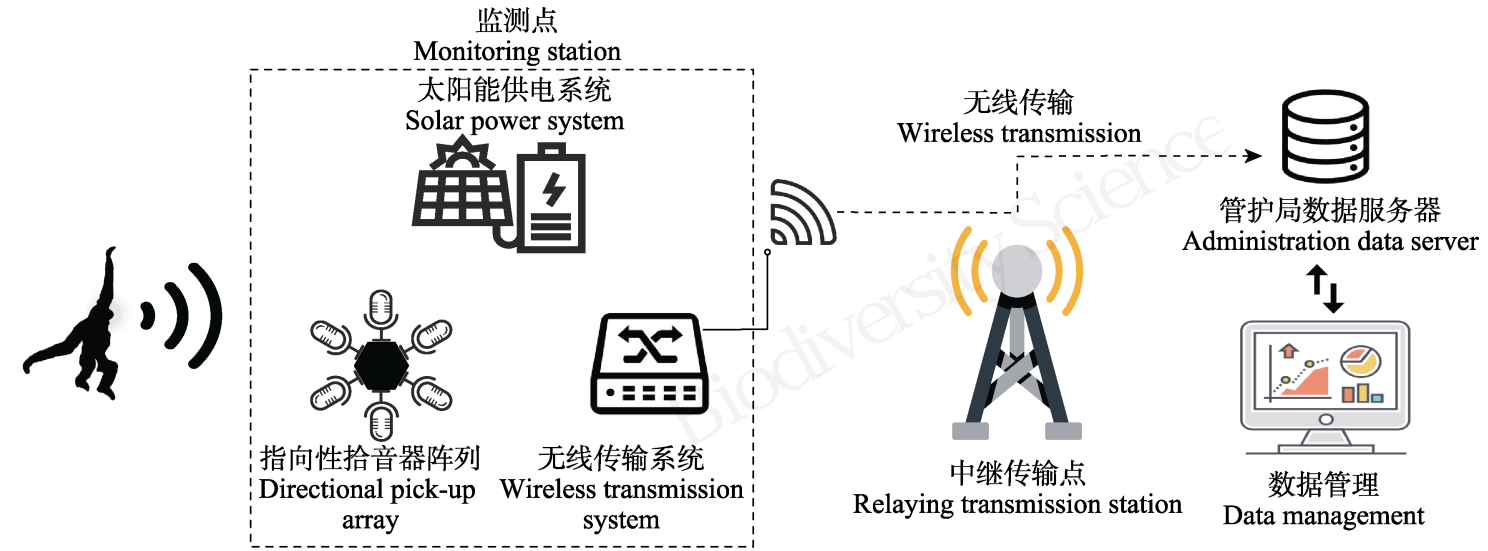

图1 被动声学监测系统架构

Fig. 1 Passive acoustic monitoring system architecture 
阳能供电系统供电, 每个传输节点分别配备有 4 块 $36 \mathrm{~V} / 250 \mathrm{~W}$ 多晶硅太阳能电池板 (太阳能板与地面 成 $25^{\circ}$ 夹角, 面朝正南方)和 4 块 $12 \mathrm{~V} / 200 \mathrm{Ah}$ 硅胶电 池，此配置可保障监测系统不间断工作7个连续阴 雨天。

(3)无线传输系统

监测数据通过无线数字微波传输。传输系统搭 建时, 先利用ArcGIS 10.3平台与哀牢山保护区楚雄 片区2 $\mathrm{m}$ 分辨率数字高程数据、野生动物监测点、 监测样线矢量数据进行信号传输可视化分析, 预先 确定监测网络各传输节点位置, 经实地传输测试后 确认最终传输节点位置(图3)。

图3a所示信号传输线路全长 $14.7 \mathrm{~km}$, 细分为
管护局至防火瞭望塔(传输距离: $1.5 \mathrm{~km}) 、$ 防火瞭望 塔至中继传输点(传输距离: $11.4 \mathrm{~km}$ )、中继传输点至 枇杷箐监测点(传输距离: $1.8 \mathrm{~km}$ )三个传输分段。在 民用无线数字微波频段中, $5.8 \mathrm{GHz}$ 频段雨衰小，稳 定性高, 相较于 $2.4 \mathrm{GHz}$ 频段更适合用于户外远距 离无线传输，故系统采用 $5.8 \mathrm{GHz}$ 频段户外千兆无 线网桥进行数据传输(最高传输速率: $867 \mathrm{Mbps}$ ), 无线网桥安装在监测塔顶部高度 $8 \mathrm{~m}$ 的位置。

\section{(4)鸣声数据管理系统}

为方便数据管理和处理, 本研究采用 $\mathrm{B} / \mathrm{S}$ (汶览 器/服务器架构)设计了Web端的数据管理系统, 研 究人员可直接通过此系统对鸣声数据进行管理和 初步处理。数据管理系统使用Python作为后端开发
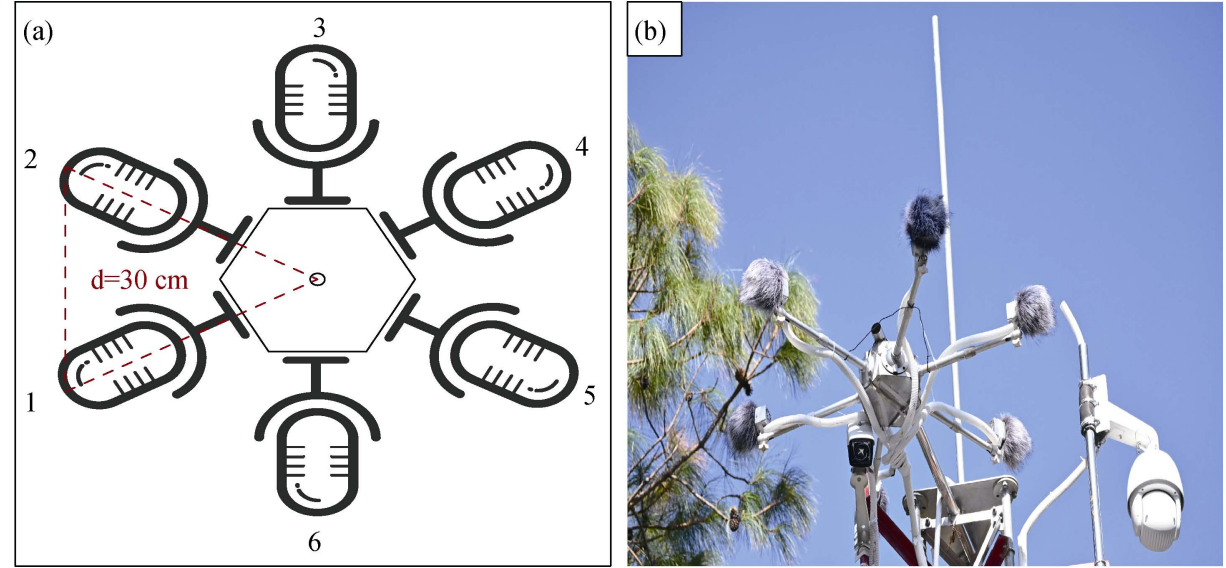

图2 指向性拾音器阵列。(a)示意图; (b)实物图。

Fig. 2 The directional pick-up array. (a) Schematic diagram; (b) Picture of real products.

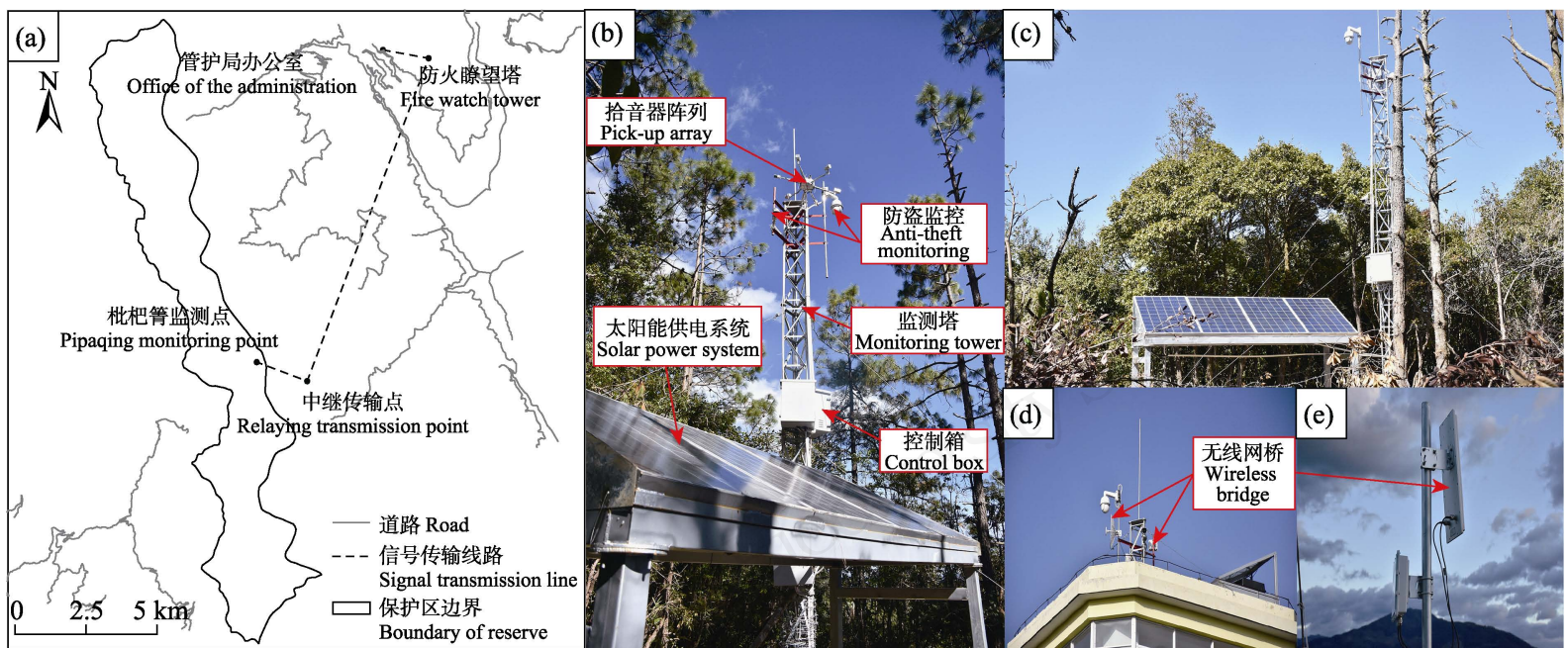

图3 无线传输线路及实物图。(a)传输线路示意; (b)监测点; (c)中继点; (d)防火瞭望塔; (e)管护局办公室。

Fig. 3 Wireless transmission line and physical figure. (a) Transmission lines diagram; (b) Monitoring point; (c) Relaying transmission point; (d) Fire watch tower; (e) Office of the administration. 
语言, 配合Flask框架和WaveSurfer.js语音插件包完 成, 具体功能包含: (1)数据管理。通过数据管理系 统访问存储于数据服务器的监测数据, 对监测数据 进行初步预览，标记含有西黑冠长臂猿鸣声的文件， 剔除无效文件。(2)数据标注。使用者在数据管理系 统中通过频谱可视化对音频文件进行标注(标注内 容包含: 起止时间、持续时间和鸣叫类型等), 并根 据需求截取存储对应音频片段。

\section{3 实验验证}

监测系统开始运行时间为2018年9月15日，实 验选取监测系统在2018年9月15日至2019年8月31 日间西黑冠长臂猿的监测结果对系统进行评估。首 先, 分析记录监测周期内采集到的西黑冠长臂猿鸣 声起止时间及鸣叫类型。其次, 在监测点西黑冠长 臂猿群体调查结果的基础上(Zhou et al, 2019), 借 助拾音器阵列对西黑冠长臂猿鸣叫方向进行辨认 (为减少误判, 仅选取两只或两只以上拾音器在同 一时刻记录到的长臂猿鸣声作为分析对象), 辨认
时先对相同时刻来自不同拾音器的鸣声进行声强 分析(Praat V6.1.09), 后将声强分析结果与拾音器空 间位置对应，进而分辨长臂猿鸣叫方向，具体判定 流程如图4所示。

\section{2 结果}

\section{1 监测结果}

被动声学监测系统从2018年9月15日至2019年 8月31日连续工作351 d, 期间共有91 d监测到西黑 冠长臂猿鸣叫(图5), 按照两次鸣叫之间 $15 \mathrm{~min}$ 及以 上间隔计算, 共监测到96次鸣叫。监测数据汇总显 示, 2019年5月是监测周期内西黑冠长臂猿鸣叫最 多的月份，单月共有19 d监测到西黑冠长臂猿鸣叫; 2019年1月与 2 月, 每月均仅有 $2 \mathrm{~d}$ 监测到西黑冠长 臂猿鸣叫, 是监测周期内西黑冠长臂猿鸣叫最少的 月份(2018年11月因供电故障，未监测到西黑冠长 臂猿鸣声)。

监测周期内监测点附近西黑冠长臂猿鸣叫开

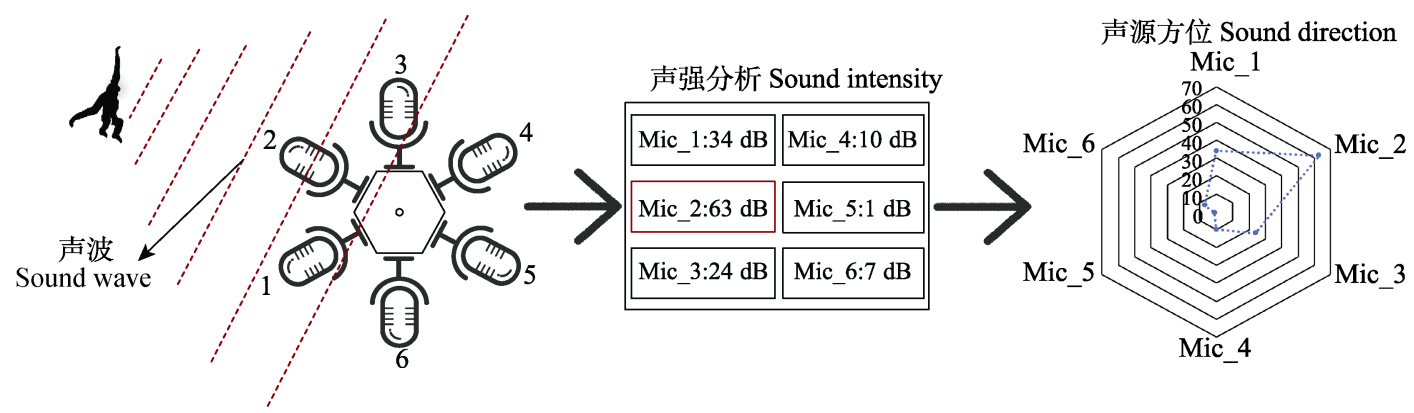

图4 鸣叫方向辨认过程

Fig. 4 Call direction recognition process

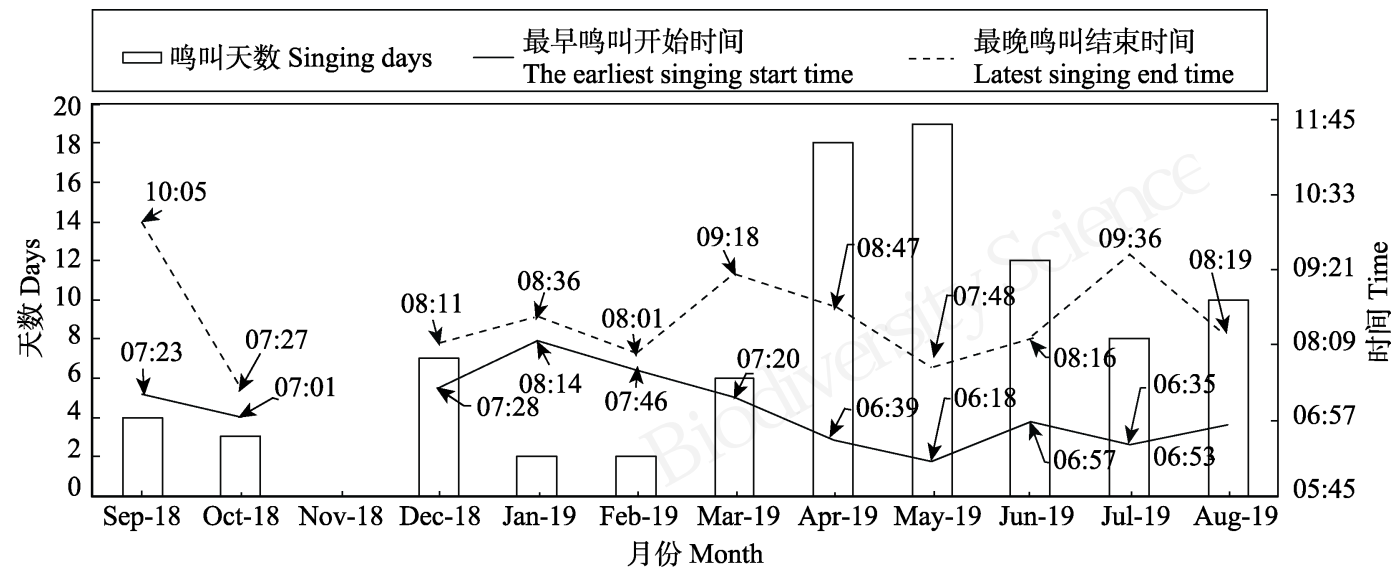

图5 2018年9月15日至2019年8月31日西黑冠长臂猿鸣声监测结果统计

Fig. 5 Statistics of monitoring results of western black crested gibbon song from September 15, 2018 to August 31, 2019 
始时间最早的是06:18 (2019年5月), 最晚的是08:14 (2019年1月), 结束时间最早的是 07:27 (2018年10 月), 最晚的是10:05 (2018年9月) (图5)。96次鸣叫平 均持续时间13.11 $\mathrm{min}$ (range: 1-37 min, $\mathrm{SD}=6.21, n$ $=96$ ), 最长持续鸣叫发生在 2019 年 4 月 4 日的 6:53-7:30, 鸣叫持续了 $37 \mathrm{~min}$ 。在2019年3月15日、 5 月22日、5月26日、6月23日和8月26日同一天内出 现了两次鸣叫。监测过程中记录到的长臂猿鸣声按 照范朋飞等(2010)对无量山西黑冠长臂猿鸣声音节 的划分方法进行划分, 包含雄性简单的重复音节 (aa notes) (图6a, b)、弱调节音节(weakly modulated figures) (图6c，d)、强调节音节(modulated figures) (图6e, f)和雌性激动鸣叫(great call) (图7)。

\section{2 鸣叫方向辨认结果}

监测周期内, 拾音器阵列中两只或两只以上拾 音器同时记录到西黑冠长臂猿鸣声的情况共有 $29 \mathrm{~d}$,
占有鸣声记录总天数的 $31.8 \%$ (共91 d)。仅有单只拾 音器记录到长臂猿鸣声的共有 $62 \mathrm{~d}$, 其中 2 号拾音 器共记录 $59 \mathrm{~d}$, 占有鸣声记录总数的 $64.8 \%$ (共91 d), 1 号拾音器共记录 $2 d, 3$ 号拾音器记录 $1 d$, 其余的 4、5、6号拾音器未出现过单独记录到长臂猿鸣声 的情况。

监测点附近西黑冠长臂猿群体鸣声聚类(提取 长臂猿鸣声的MFCC特征, 使用Mean Shift算法, 聚 类过程见Zhou et al, 2019)和实地调查结果显示, 监 测点附近有 2 个西黑冠长臂猿群体。而拾音器阵列 中的 6 个拾音器均能记录到群体 1 和群体 2 的鸣声。 将监测点不同长臂猿群体鸣声聚类结果与拾音器 阵列的声强分析结果(表1)、鸣声方向分辨结果(图 8)对照分析后发现，西黑冠长臂猿群体1的鸣声主 要来自 2 号拾音器所指方向, 声强最大值为 65.88 $\mathrm{dB}$ 。群体2的鸣声主要来自3、4号拾音器所指方向,
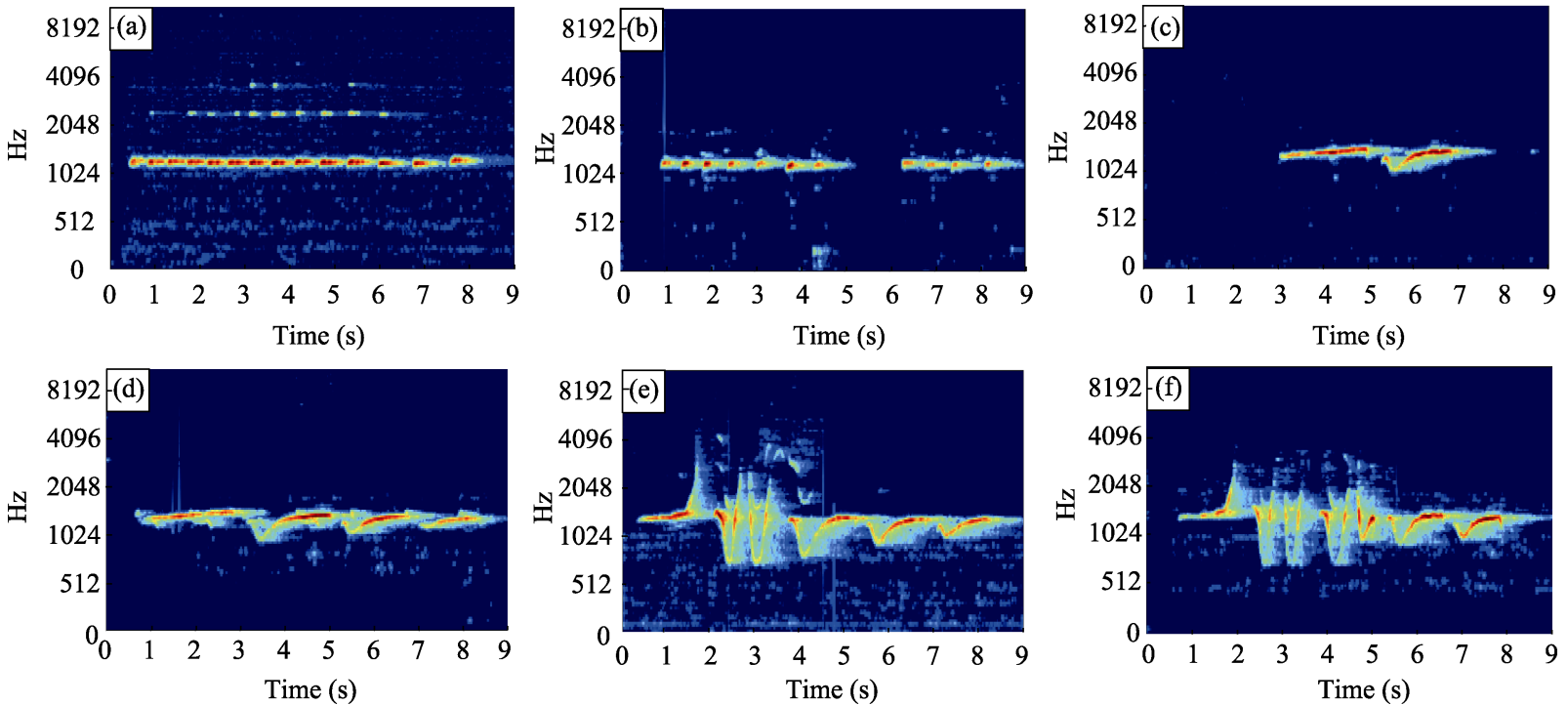

图6 监测点附近成年雄性西黑冠长臂猿不同鸣叫类型的声谱图。a, b: 重复音节; c, d: 弱调节音节; e, f: 强调节音节。

Fig. 6 The sonogram of adult western black crested gibbon male’s call types in monitoring station. a, b: Aa notes; c, d: Weakly modulated figures; e, f: Modulated figures.
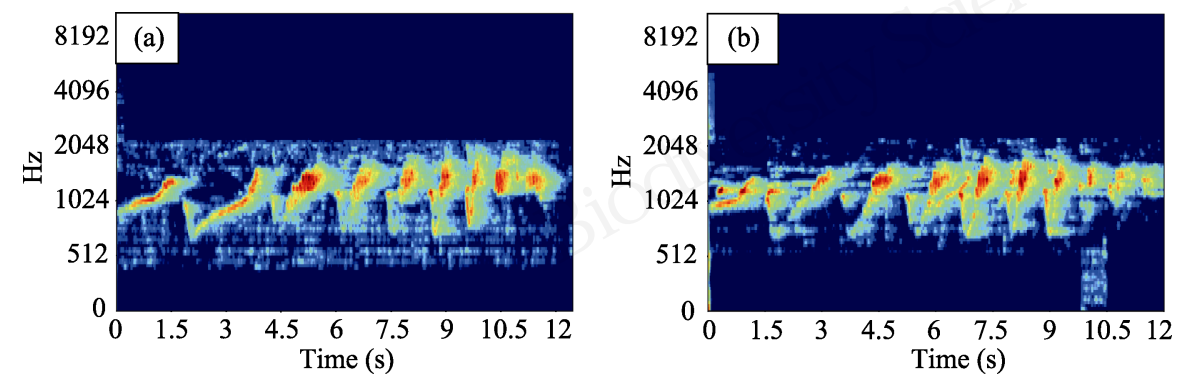

图7 监测点附近雌性西黑冠长臂猿不同类型激动鸣叫的声谱图

Fig. 7 The sonogram of adult western black crested gibbon female’s great call types in monitoring station 
表1＼cjkstart鸣声样本声强检测结果及对应西黑冠长臂猿群体

Table 1 Sound intensity detection results of the call samples and corresponding population of the western black crested gibbon

\begin{tabular}{llllllll}
\hline \multirow{2}{*}{ 日期 Date } & \multicolumn{9}{c}{ 鸣声声强 } & Intensity of calls (dB) & \multicolumn{2}{c}{ No.6 } & 群体 Group \\
\cline { 2 - 7 } & No.1 & No.2 & No.3 & No.4 & No.5 & G. \\
\hline $2018 / 9 / 17$ & 9.438 & 64.29 & 42.22 & 40.84 & 13.17 & 39.2 & G1 \\
$2018 / 9 / 20$ & - & 53.22 & 57.47 & 63.77 & 11.96 & 56.21 & G2 \\
$2018 / 10 / 22$ & - & 57.87 & 24.36 & - & - & - & G1 \\
$2019 / 5 / 7$ & - & 43.41 & 39.02 & 31.91 & 10.38 & - & G1 \\
$2019 / 5 / 8$ & - & 25.44 & 56.75 & 34.49 & - & - & G2 \\
$2019 / 5 / 23$ & 8.7 & 65.88 & 43.07 & 41.66 & - & - & G1 \\
$2019 / 6 / 25$ & - & 58.54 & - & 38.68 & 30.9 & - & G1 \\
$2019 / 6 / 29$ & - & - & 38.62 & 56.87 & - & - & G2 \\
\hline
\end{tabular}
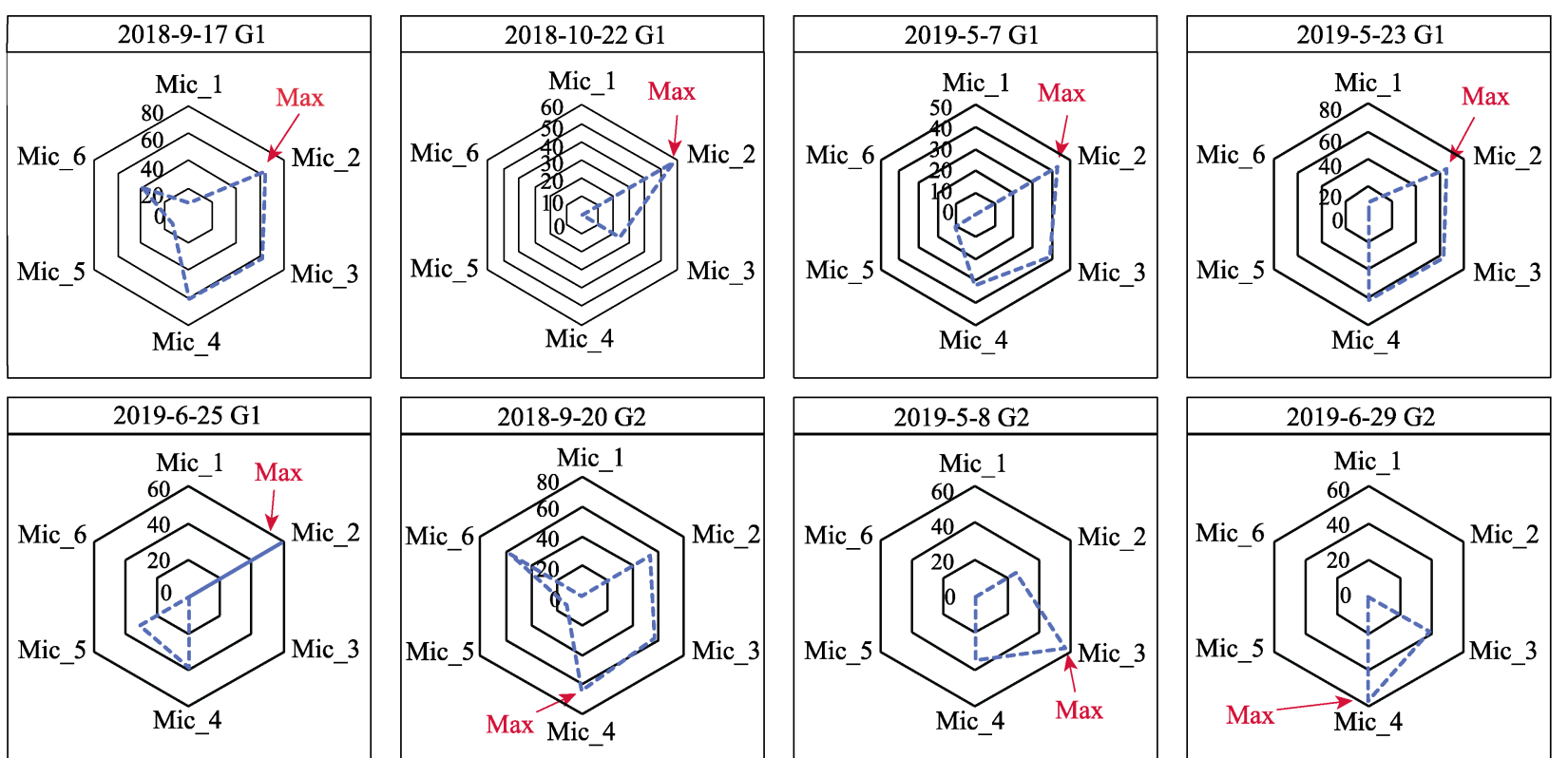

图8 监测点附近西黑冠长臂猿鸣叫方向示意

Fig. 8 Call directions of the western black crested gibbon in monitoring station

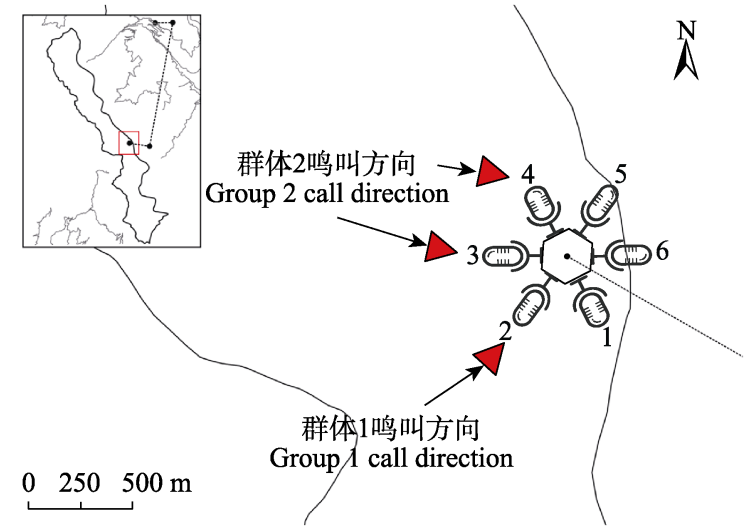

图9 监测点附近西黑冠长臂猿鸣叫方向

Fig. 9 Call directions of the western black crested gibbon in monitoring station
其中3号拾音器对应最大鸣声强度为 $56.75 \mathrm{~dB}, 4$ 号 拾音器对应的最大鸣声强度为 $63.77 \mathrm{~dB}$ 。

对照拾音器阵列实际安装位置(图9)可见, 群 体1主要分布在枇杷等监测点西南方向, 群体2主要 分布在监测点西北方向, 拾音器阵列方向分辨结果 与护林员实际调查结果一致。在2018年9月至2019 年6月间拾音器阵列记录到的长臂猿鸣声始终对应 着2 (群体1), 3、4 (群体2)号拾音器所指方向, $1 、 5$ 、 6 号拾音器所指方向从未单独记录到任意一个西黑 冠长臂猿群体的鸣声, 这表明监测点附近的两个西 黑冠长臂猿群体领地相邻, 而不同时间点两个长臂 猿群体的鸣声声强变化也表明西黑冠长臂猿鸣叫 
地点并不固定。

\section{3 讨论}

长臂猿监测工作一般从鸣声监测开始, 这是获 得长臂猿种群信息最直接、最有效的手段, 需贯穿 整个监测周期(管振华等, 2017)。本研究的目的是开 发一套自动化的被动声学监测系统, 并将其应用到 西黑冠长臂猿的长期监测工作中。该系统使用太阳 能供电, 并通过无线微波实时传输数据。系统最大 的特点在于使用自研的指向性拾音器阵列对西黑 冠长臂猿鸣声进行监测, 除了能记录长臂猿鸣声之 外, 还能分辨长臂猿鸣声来源方向。监测数据实时 传回管护局存储在数据服务器中, 研究人员可借助 后台数据管理系统对数据进行管理分析。实验结果 表明, 本研究设计的被动声学监测系统长期运行稳 定, 拾音器阵列能有效分辨西黑冠长臂猿鸣声来源 方向, 适用于西黑冠长臂猿的长期监测。

本文设计的自动化被动声学监测系统与传统 人工监测方法相比最大的优势在于, 系统使用太阳 能供电并配合无线网络进行实时传输, 无需定期进 行电池及存储介质的更换, 有效降低了后期管理成 本, 减少数据损坏丢失的风险。同时, 系统可长期 连续运行的特点使得研究者可获得持续性更好、完 整度更高的监测数据。而指向性拾音器阵列的应用, 也为研究人员在鸣声监测时能有效分辨西黑冠长 臂猿鸣声来源方向提供了参考。

本文设计的被动声学监测系统作为一种新的 监测手段, 在未来推广应用时需从成本、性能和稳 定性三个方面来综合考虑。从监测成本来看, 系统 使用的拾音器阵列成本约为 1 万元人民币, 太阳能 供电系统和无线传输系统以及各项基础费用约为 2 万元人民币。同时, 系统使用自己搭建的无线网络, 无需额外支付网络通信费用, 按照至少 5 年的使用 寿命计算, 平均每年花费约为 0.6 万元。从长期监测 的角度来看, 它比不带有太阳能或远程数据传输功 能的声学监测设备(例如Wildlife Acoustic Song Meter 4, 约849美元/台)或商业化的监测系统(例如 ARBIMON (Aide et al, 2013), 约3千美元/年但不包 含监测硬件)的性价比更高。

从指向性拾音器阵列的监测范围来看, 我们在 距离监测点1.8 km左右的中继点, 使用牛角号(楚雄 当地传统乐器, 鸣奏时频率集中在1-3 kHz, 声强约
为80-100 dB)进行了测试, 指向性拾音器阵列能清 晰记录到牛角号的号声。而西黑冠长臂猿鸣声的主 频率也主要集中在1-2 kHz左右, 传播距离在 $2 \mathrm{~km}$ 左右(范朋飞等, 2010), 故可以大致估算得到指向 性拾音器阵列的监测范围约为以监测点为圆心半 径 $2 \mathrm{~km}$ 左右的范围。就哀牢山国家级自然保护区楚 雄片区这样狭长的区域而言(楚雄片区长约 $20 \mathrm{~km}$, 最宽处约 $4.2 \mathrm{~km})$, 大约5-8个监测点便可对片区内 西黑冠长臂猿实现覆盖式监测。

监测系统从2018年9月15日起开始运行, 就监 测系统设备运行情况来说, 系统内设备均未出现过 硬件故障。但从监测记录统计结果(图5)来看, 2018 年10月至2019年2月间每月记录到鸣声的天数少于 2019年3月至8月记录到的鸣叫天数。除了因为季节 造成的西黑冠长臂猿鸣叫频次差异外, 我们在对系 统运行日志分析后发现，造成2018年9月至2019年2 月监测结果较少的原因还包括以下两个方面: (1)监 测初期系统整体运行还未完全稳定, 监测系统数据 传输网络的临时故障、管护局停电造成的数据服务 器暂停工作等原因均会造成监测数据的不连续; (2) 长臂猿鸣叫主要发生在日出前 $0.5 \mathrm{~h}$ 至日出后 $3 \mathrm{~h}$ 内 (范朋飞等, 2010), 通常这个时间段监测设备仅依靠 蓄电池供电, 超过 7 天的连续阴雨天和太阳能板周 围树木阴影的遮挡会造成太阳能供电系统发电效 率较低, 蓄电池储备电量无法在清晨完全满足系统 运行需求。仅当 10 点以后温度回升太阳照射面积加 大太阳能板开始工作, 系统才有足够的电力供应以 恢复运行, 而此时西黑冠长臂猿的鸣叫大多已经结 束, 即使记录到也仅有一小段。为保证监测系统运 行的稳定性和数据的完整性, 我们为监测系统内各 子系统增加了智能控制开关，通过控制开关可设定 各子系统工作状态及时长, 合理调配电能使用情况, 以保证系统在长臂猿鸣叫集中的时间段始终正常 工作。通过对系统的调整和改进, 监测系统从2019 年3月开始便一直正常运行未再出现过监测中断的 情况。不过, 长臂猿鸣声所在的1-2 kHz频段附近还 有竹鸡 (Bambusicola fytchii)、府鸪 (Francolinus pintadeanus)等鸟类鸣声会与长臂猿鸣声混叠, 造 成后期数据处理困难, 这也是未来被动声学监测系 统推广应用过程中, 需重点关注的问题。

在未来的工作中, 此系统可向小型化和便携化 方向发展, 进一步降低搭建成本, 并结合声音事件 
识别、大数据和机器学习等技术实现智能化监测, 方便研究者将其与原有监测方法结合互补, 从不同 的时空尺度构建更大规模的监测网络。同时, 此系 统改进后也适用于具有大声鸣叫行为的其他动物 类群的监测或是配合多种传感器用于更广泛的生 物多样性监测。

\section{ORCID}

钟恩主 (D) https://orcid.org/0000-0002-3927-0927

管振华 (iD https://orcid.org/0000-0003-4613-9529

胡坤融 (D) https://orcid.org/0000-0001-8847-4873

致谢：本研究得到哀牢山国家级自然保护区楚雄分 局的大力支持和协助!

\section{参考文献}

Aide TM, Corrada-Bravo C, Campos-Cerqueira M (2013) Real-time bioacoustics monitoring and automated species identification. PeerJ, 1, e103.

Blumstein DT, Mennill DJ, Clemins P, Girod L, Yao K, Patricelli G, Deppe JL, Krakauer AH, Clark C, Cortopassi KA, Hanser SF, McCowan B, Ali AM, Kirschel ANG (2011) Acoustic monitoring in terrestrial environments using microphone arrays: Applications, technological considerations and prospectus. Journal of Applied Ecology, 48, 758-767.

Brockelman WY, Srikosamatara S (1993) Estimation of density of gibbon groups by use of loud songs. American Journal of Primatology, 29, 93-108.

Chesmore ED, Ohya E (2004) Automated identification of field-recorded songs of four British grasshoppers using bioacoustic signal recognition. Bulletin of Entomological Research, 94, 319-330.

Enari H, Enari H, Okuda K, Yoshita M, Kuno T, Okuda K (2017) Feasibility assessment of active and passive acoustic monitoring of sika deer populations. Ecological Indicators, 79, 155-162.

Fan PF, Jiang XL, Liu CM, Luo WS (2010) Sonogram structure and timing of duets of western black crested gibbon in Wuliang Mountain. Zoological Research, 31, 293-302. (in Chinese with English abstract) [范朋飞, 蒋学 龙, 刘长铭, 罗文寿 (2010) 无量山西黑冠长臂猿二重唱 的声谱结构和时间特征. 动物学研究, 31, 293-302.]

Fan PF, Xiao W, Huo S, Jiang XL (2009) Singing behavior and singing functions of black-crested gibbons (Nomascus concolor jingdongensis) at Mt. Wuliang, central Yunnan, China. American Journal of Primatology, 71, 539-547.

Guan ZH, Yan L, Huang B (2017) Population monitoring of gibbons in China. Sichuan Journal of Zoology, 36, 232-238. (in Chinese with English abstract) [管振华, 阎璐, 黄蓓 (2017) 中国长臂猿科动物种群监测现状分析. 四川动物,
36, 232-238.]

IUCN (2020) The IUCN Red List of Threatened Species. Version 2020-1. https://www.iucnredlist.org. (accessed on 2020-03-19)

Kalan AK, Mundry R, Wagner OJ, Heinicke S, Boesch C, Kühl HS (2015) Towards the automated detection and occupancy estimation of primates using passive acoustic monitoring. Ecological Indicators, 54, 217-226.

Kalan AK, Piel AK, Mundry R, Wittig RM, Boesch C, Kühl HS (2016) Passive acoustic monitoring reveals group ranging and territory use: A case study of wild chimpanzees (Pan troglodytes). Frontiers in Zoology, 13, 1-11.

MacSwiney GMC, Clarke FM, Racey PA (2008) What you see is not what you get: The role of ultrasonic detectors in increasing inventory completeness in Neotropical bat assemblages. Journal of Applied Ecology, 45, 1364-1371.

McCowan I (2001) Robust Speech Recognition Using Microphone Arrays. PhD dissertation, Queensland University of Technology, Brisbane.

Swiston KA, Mennill DJ (2009) Comparison of manual and automated methods for identifying target sounds in audio recordings of pileated, pale-billed, and putative ivory-billed woodpeckers. Journal of Field Ornithology, 80, 42-50.

Sun GZ, Bei H, Geissmann T, Jiang XL (2011) Individuality in male songs of wild black crested gibbons (Nomascus concolor). American Journal of Primatology, 73, 431-438.

Sun GZ, Ni QY, Huang B, Guan ZH, Li XP, Jiang XL (2012) The population number, distribution and current situation of the western black crowned gibbon. Forestry Construction, (1), 38-44. (in Chinese) [孙国政, 倪庆永, 黄蓓, 管振华, 李小平, 蒋学龙 (2012) 西黑冠长臂猿的种群数量、分布 与现状. 林业建设, (1), 38-44.]

Spillmann B, Maria VN, Willems EP, Setia TM, Wipfli U, Schaik CPV (2015) Validation of an acoustic location system to monitor Bornean orangutan (Pongo pygmaeus wurmbii) long calls. American Journal of Primatology, 77, 767-776.

Thompson ME, Schwager SJ, Payne KB (2010) Heard but not seen: An acoustic survey of the African forest elephant population at Kakum Conservation Area, Ghana. African Journal of Ecology, 48, 224-231.

Watkins WA, Schevill WE (1972) Sound source location by arrival-times on a non-rigid three-dimensional hydrophone array. In: Deep Sea Research and Oceanographic Abstracts, pp. 691-706. Elsevier, Woods Hole.

Zhou XC, Guan ZH, Zhong EZ, Dong YY, Li H, Hu KR (2019) Automated monitoring of western black crested gibbon population based on voice characteristics. In: 2019 IEEE 5th International Conference on Computer and Communications, pp. 1383-1387. IEEE, Chengdu.

(责任编委: 乔慧捷 责任编辑: 问文杰) 